\title{
Genome-wide identification and Phylogenic analysis of kelch motif containing ACBP in Brassica napus
}

Nadia Haingotiana Raboanatahiry ${ }^{1,2}$, Yongtai Yin ${ }^{1,2}$, Li Chen ${ }^{1,2}$ and Maoteng $\mathrm{Li}^{1,2^{*}}$

\begin{abstract}
Background: Acyl-COA binding proteins (ACBPs) bind long chain acyl-CoA esters with very high affinity. Their possible involvement in fatty acid transportation from the plastid to the endoplasmic reticulum, prior to the formation of triacylglycerol has been suggested. Four classes of ACBPs were identified in Arabidopsis thaliana: the small ACBPs, the large ACBPs, the ankyrin repeats containing ACBPs and the kelch motif containing ACBPs. They differed in structure and in size, and showed multiple important functions. In the present study, Brassica napus ACBPs were identified and characterized.

Results: Eight copies of kelch motif ACBPs were cloned, it showed that B. napus ACBPs shared high amino acid sequence identity with A. thaliana, Brassica rapa and Brassica oleracea. Furthermore, phylogeny based on domain structure and comparison map showed the relationship and the evolution of ACBPs within Brassicaceae family: ACBPs evolved into four separate classes with different structure. Chromosome locations comparison showed conserved syntenic blocks.

Conclusions: ACBPs were highly conserved in Brassicaceae. They evolved from a common ancestor, but domain duplication and rearrangement might separate them into four distinct classes, with different structure and functions. Otherwise, B. napus inherited kelch motif ACBPs from ancestor conserving chromosomal location, emphasizing preserved synteny block region. This study provided a first insight for exploring ACBPs in B. napus, which supplies a valuable tool for crop improvement in agriculture.
\end{abstract}

Keywords: Acyl-coA binding proteins, kelch motif, Brassica, Evolution, Phylogeny, Chromosome map

\section{Background}

Acyl-coA binding proteins (ACBPs) bind long chain acylcoA esters through an acyl-coA-binding domain, with very high affinity [1-3]. They are involved in the formation of long-chain fatty acyl-coA pools and act as an intracellular acyl-coA transporter [4-6]. ACBPs are possibly the transporters of synthesized fatty acids from plastid to endoplasmic reticulum, prior to the biosynthesis of triacylglycerol (TAG), principal compound of essential oils used for food, biofuel and many industrial applications. Arabidopsis thaliana ACBPs (AtACBPs) are divided in four classes, with six members that differ in structure, functions,

\footnotetext{
* Correspondence: limaoteng426@mail.hust.edu.cn

${ }^{1}$ College of Life Science and Technology, Huazhong University of Science and Technology, Wuhan 430074, China

${ }^{2}$ Hubei Collaborative Innovation Center for the Characteristic Resources Exploitation of Dabie Mountains, Huanggang 435599, China
}

expression, subcellular location and acyl-coA ester affinities $[1,2,7-10]$. Class I are the smallest ACBPs, usually called ACBP6, and contain 92 amino acids. ACBP6 are localized in the cytosol, where they have affinity to bind linoleyl-coA [10], they are expressed in all plants organs [11]. These small ACBPs are involved in intracellular binding and trafficking of phosphatidylcholine in plant phospholipid metabolism [11]. They maintain balance between acyl-coA and phosphatidylcholine to improve acyl exchange [12]. Class II correspond to the ankyrin repeats proteins (ACBP1 and ACBP2); they share the $76.9 \%$ of identity and have respectively 338 and 355 amino acids. ACBP1 and ACBP2 are localized in the plasma membrane and in the endoplasmic reticulum [10], both of them are membrane proteins involved in membraneassociated acyl-coA transfer and metabolism functions [13], they bind linoleoyl-CoA and linolenoyl-CoA esters 
[14]. ACBP1 are highly expressed in seeds and siliques [7] and ACBP2 are expressed in roots, stems and flowers [15]. The class III ACBPs are commonly called "large ACBPs" or ACBP3, they contain 362 amino acids. ACBP3 have an extracellular location, they are highly expressed in siliques and young shoots [10]. ACBP3 bind arachidonyl-CoA with high affinity [2]. The kelch motif proteins are the class IV ACBPs (ACBP4 and ACBP5); these two copies share the $81.4 \%$ of identity and have respectively 668 and 648 amino acids. ACBP4 and ACBP5 are located in the cytosol [10], where they have high affinity to bind oleoyl-coA. In presence of free oleoyl-coA that might inhibit the glucose-6-phosphate in the plastid, they can suppress synthesis dysfunction of starch and fatty acid [1]. They have more significant role in lipid transfer from the plastids to the endoplasmic reticulum $[11,16]$, and more roles for the biosynthesis of non-plastidial membrane lipids [17]. These kelch motif ACBPs are expressed in all tissues, with a higher expression of ACBP4 in roots and a lower expression in siliques. ACBP5 were found in young shoots and mature leaves but in a lower rate than ACBP4 [18].

Brassica napus is an important oil crop. It is a descendant lineage from hybridization between Brassica rapa and Brassica oleracea. B. napus 10-kDa ACBPs (BnACBPs) were identified twenty years ago [19]. Recent study revealed that they could change acyl-coA and TAG composition in seeds [20]. The kelch motif AtACBPs have many important functions. Besides their ability to maintain and transport oleoyl-coA from plastid to endoplasmic reticulum in plant metabolism, their structure that contains kelch domains, a protein-protein interaction site, makes them involved in plant stress responses $[13,18,21$, 22 . Due to the importance attributed to the kelch motif AtACBPs and those of small BnACBPs, it might be possible that the kelch motif $B n A C B P s$ share the same, or more, functions with them. Thus, the identification and characterization of $B n A C B P s$ could provide a basis for further studies about their functions. In this way, their exploitation can be useful in agriculture.

The present study aims to explore ACBPs in B. napus. This report contains four parts. Firstly, ACBPs were identified and characterized in B. rapa, B. oleracea and B. napus; amino acid sequence identities were compared. Secondly, AtACBPs and BnACBPs domain structures were analyzed. Thirdly, a phylogenetic analysis based on conserved domain was carried out. Finally, the comparison in chromosome locations of the kelch motif ACBPs was made.

\section{Results}

ACBPs in B. rapa, B. oleracea and B. napus

ACBPs were identified based on homology with AtACBPs. Similar to AtACBPs, they could be divided in four classes, with multiple copies (Table 1). Small ACBPs had 90 to 92 amino acids, ankyrin repeats ACBPs had 339 to 364 amino acids, large ACBPs had 361 to 381 amino acids and kelch motif ACBPs had 665 to 667 amino acids. Small ACBPs in B. rapa (BrACBPs) and in $B$. oleracea (BoACBPs) had three copies each; however, small $B n A C B P s$ had only four copies. They were inherited from Bra033875 (BnaA05g36060D), Bra038439 (BnaA08g07670D), Bra023206 (BnaAnng25690D) and Bol038626 (BnaCnng15340D). Amino acid sequence comparison showed that small BnACBPs could share an average identity of $82.6 \%$ with AtACBPs, $99.6 \%$ with BrACBPs, and $98.5 \%$ with B. oleracea ACBPs BoACBPs (Table 2). Each of ankyrin repeats BrACBPs and BoACBPs had two copies. Thus, four ankyrin repeats BnACBPs could share about $85.5 \%$ of amino acid sequence identity with AtACBPs, $98.3 \%$ with BrACBPs, and $98.9 \%$ with $B o A C B P s$. In addition, two ankyrin repeats $B n A C B P s$ (BnaA02g10270D and BnaC02g44810D) might belong to ACBP1 subclass ( $86.8 \%$ of identity with AtACBP1), and two other ankyrin repeats BnACBPs (BnaA01g16660D and BnaC01g20440D) might be ACBP2 ( 85.9 \% of identity with AtACBP2). Four copies of large BnACBPs were inherited from four copies of large ACBPs of $B$. rapa and B. oleracea. They shared about $59.7 \%$ of amino acid sequence identity with AtACBPs, $98.5 \%$ with BrACBPs, and $94.1 \%$ with BoACBPs. Eight copies of kelch motif $B n A C B P s$ were cloned and sequenced. They are available on GenBank (accession: AIS76194 to AIS76201). Kelch motif $B n A C B P s$ were cloned from material grown in a semi-winter type area in China, they were the subject to further function analysis; but also, different lines SNP might exist as the material used for the sequencing of $B$. napus is not the same. These kelch motif BnACBPs were compared with six copies of putative kelch motif $B n A C B P s$ found in the database. Amino acid sequence identity was compared (Table 3). Both AIS76195 and AIS76200 shared $99.7 \%$ of amino acid sequence identity with BnaA05g31780D, and $99.3 \%$ of identity with Bra039439. However, AIS76195 shared $99.6 \%$ of identity with AIS76200. Similarly, both AIS76196 and AIS76201 shared respectively $100 \%$ and $97.6 \%$ of identity with BnaAnng02420D, and $100 \%$ and $97.6 \%$ with Bol002733. They, however, shared $99.1 \%$ of identity between them. Besides, AIS76194 and AIS76199 shared $97.1 \%$ of identity and AIS76197 and AIS76198 shared $95.5 \%$ of identity. These eight cloned copies of kelch motif $B n A C B P s$ could share amino acid sequence identity of about $88.25 \%$ with AtACBPs, 98.17 \% with BrACBPs, and 98.4 \% with BoACBPs. Otherwise, AIS76194, AIS76195, AIS76196, AIS76199, AIS76200 and AIS76201 could be ACBP4 ( 90.53\% of identity with AtACBP4), and AIS76197 and AIS76198 could be ACBP5 ( 81.4 \% of identity with AtACBP5). These results indicated that ACBPs were conserved in B. rapa, B. oleracea and B. napus. 
Table 1 ACBP in A. thaliana, B. rapa, B. oleracea and B. napus. An, Cn or Un correspond to unspecified chromosome location

\begin{tabular}{|c|c|c|c|c|c|c|c|c|c|c|c|c|}
\hline \multirow[t]{2}{*}{ Class } & \multicolumn{3}{|l|}{ A. thaliana } & \multicolumn{3}{|l|}{ B. rapa } & \multicolumn{3}{|l|}{ B. oleracea } & \multicolumn{3}{|l|}{ B. napus } \\
\hline & & Chr & Size (Aa) & & Chr & Size (Aa) & & Chr & Size (Aa) & & Chr & Size $(\mathrm{Aa})$ \\
\hline \multirow[t]{4}{*}{ Small } & At1g31812 & AT1 & 92 & Bra033875 & A5 & 92 & Bol038626 & C4 & 92 & BnaAnng25690D & An & 90 \\
\hline & & & & Bra038439 & A8 & 92 & Bol027060 & $\mathrm{C} 8$ & 92 & BnaA05g36060D & A5 & 92 \\
\hline & & & & Bra023206 & A9 & 90 & Bol005980 & C5 & 90 & BnaA08G07670D & A8 & 92 \\
\hline & & & & & & & & & & BnaCnng15340D & $\mathrm{Cn}$ & 92 \\
\hline Ankyrin & At4g27780 & AT4 & 354 & $\mathrm{Bra026307}$ & $\mathrm{A} 1$ & 364 & Bol013113 & $\mathrm{C} 1$ & 364 & BnaA02g10270D & $\mathrm{A} 2$ & 342 \\
\hline \multirow[t]{3}{*}{ repeats } & At5g53470 & AT5 & 338 & Bra022656 & $\mathrm{A} 2$ & 341 & Bol017188 & C6 & 339 & BnaA01g16660D & A1 & 364 \\
\hline & & & & & & & & & & BnaC02g44810D & $C 2$ & 339 \\
\hline & & & & & & & & & & BnaC01g20440D & C1 & 364 \\
\hline \multirow[t]{4}{*}{ Large } & At4g24230 & AT4 & 366 & Bra013778 & $\mathrm{A} 1$ & 376 & Bol009564 & $\mathrm{C} 1$ & 370 & BnaA01g13710D & A1 & 381 \\
\hline & & & & Bra019240 & A3 & 362 & Bol042158 & $\mathrm{C} 6$ & 361 & BnaA03g46540D & A3 & 362 \\
\hline & & & & & & & & & & BnaC01g16110D & C1 & 364 \\
\hline & & & & & & & & & & BnaC07g38820D & C7 & 361 \\
\hline Kelch & At3g05420 & AT3 & 668 & Bra020582 & $\mathrm{A} 2$ & 667 & Bol012774 & $\mathrm{C} 2$ & 667 & AIS76194 & A3 & 666 \\
\hline \multirow[t]{14}{*}{ motif } & At5g27630 & AT5 & 648 & Bra001147 & A3 & 666 & Bol001638 & C5 & 667 & (BnaA03g29000D) & & \\
\hline & & & & Bra039439 & A5 & 667 & Bol002733 & $\mathrm{C} 1$ & 665 & AIS76195 & A5 & 667 \\
\hline & & & & Bra040219 & An & 665 & Bol034106 & C3 & 666 & (BnaA05g31780D) & & \\
\hline & & & & & & & & & & AIS76196 & An & 665 \\
\hline & & & & & & & & & & (BnaAnng02420D) & & \\
\hline & & & & & & & & & & AIS76197 & $\mathrm{C} 2$ & 667 \\
\hline & & & & & & & & & & (BnaC02g39790D) & & \\
\hline & & & & & & & & & & AIS76198 & $\mathrm{A} 2$ & 667 \\
\hline & & & & & & & & & & (BnaA02g31230D) & & \\
\hline & & & & & & & & & & AIS76199 & C3 & 666 \\
\hline & & & & & & & & & & (BnaC03g34240D) & & \\
\hline & & & & & & & & & & AIS76200 & A5 & 667 \\
\hline & & & & & & & & & & (BnaA05g31780D) & & \\
\hline & & & & & & & & & & AIS76201 & Un & 665 \\
\hline
\end{tabular}

Moreover, $B n A C B P s$ shared high amino acid sequences identity with AtACBPs, BrACBPs and BoACBPs.

\section{Domain structure of AtACBPs and BnACBPs}

Domain structure of AtACBPs and BnACBPs were analyzed (Fig. 1). They commonly contained the acyl-coA binding domain (ACBD), but obviously differed in ACBD location and in extra domain structures in two classes of ACBP, the ankyrin repeats and the kelch motif ACBPs. ACBD was the only domain apparent in small ACBPs and seemed to be extended in almost all the protein (residue 3 to 87). Ankyrin repeats ACBPs had N-terminal ACBD (residue 112-182 in ACBP1 and 101-192 in ACBP2), and C-terminal ankyrin domain (residue 221-328 in ACBP1 and 236-349 in ACBP2). Similar to small ACBPs, large ACBPs had only one domain corresponding to ACBD (residue 231-325) located in the C-terminal of the proteins. However,
ACBD were located in the $\mathrm{N}$-terminal side of kelch motif ACBPs (residue 14-104 in ACBP4 and 12-105 in ACBP5). Additionally, four or five kelch motifs that belonged to Kelch 3 superfamily were found. Alignment of each kelch motif showed 40 highly conserved amino acid residues in the first kelch motif (181-226 in ACBP4, 179-220 in ACBP5), 29 residues in the second kelch motif (242-283 in ACBP4, 243-288 in ACBP5), 38 residues in the third kelch motif (303-354 in ACBP4, 303-355 in ACBP5), 38 residues in the fourth kelch motif (357-405 in ACBP4, 357-406 in ACBP5), and 36 residues in the fifth kelch motif (392-431 in ACBP4, 395-439 in ACBP5) (see Figure S1, Additional file 1). Apparently, the second kelch motif was missing in At5g27630, in AIS76194 and in AIS76198 (see Figure S1, Additional file 1-II). Alignment showed that At5g27630 differed from other ACBPs in Gln-256, Pro-272, Met-278, Cys-280 and Ser-282, likewise in AIS76194 and AIS76198, 
Table 2 Comparison of amino acid sequence identity in AtACBPs, BrACBPs, BoACBPs and BnACBPs. Numbers are in percent (\%)

\begin{tabular}{|c|c|c|c|c|c|c|c|c|c|c|c|}
\hline \multirow{2}{*}{$\frac{\text { Class }}{\text { Small }}$} & \multirow[t]{2}{*}{ BnACBPs } & \multirow{2}{*}{\multicolumn{2}{|c|}{$\frac{\text { AtACBPs }}{\text { At1g31812 }}$}} & \multicolumn{4}{|l|}{ BrACBPs } & \multicolumn{4}{|l|}{ BoACBPs } \\
\hline & & & & Bra033875 & Bra038439 & Bra023206 & & Bol038626 & Bol027060 & Bol005980 & \\
\hline & BnaAnng25690D & 80.4 & & 83.9 & 87 & 100 & & 84.9 & 84.8 & 97.8 & \\
\hline & BnaA05g36060D & 83.7 & & 98.9 & 94.6 & 84.9 & & 97.8 & 92.4 & 84.9 & \\
\hline & BnaA08G07670D & 82.6 & & 93.5 & 100 & 87 & & 92.4 & 97.8 & 87.4 & \\
\hline & BnaCnng15340D & 83.7 & & 96.7 & 92.4 & 84.9 & & 100 & 90.2 & 84.9 & \\
\hline Ankyrin & & At5g53470 & At4g27780 & Bra026307 & & Bra022656 & & Bol013113 & & Bol017188 & \\
\hline \multirow[t]{4}{*}{ repeats } & BnaA02g10270D & 86.1 & 73.8 & 70.1 & & 98.8 & & 69.9 & & 94.7 & \\
\hline & BnaC02g44810D & 87.5 & 73.9 & 71.2 & & 95 & & 71 & & 100 & \\
\hline & BnaA01g16660D & 70.8 & 84.3 & 97.8 & & 71.8 & & 96.2 & & 72.1 & \\
\hline & BnaC01g20440D & 70.3 & 84.3 & 97.3 & & 70.9 & & 97.8 & & 71.8 & \\
\hline \multirow[t]{5}{*}{ Large } & & At4g24230 & & Bra013778 & & Bra019240 & & Bol009564 & & Bol042158 & \\
\hline & BnaA01g13710D & 62.9 & & 97.4 & & 64.9 & & 85.3 & & 64.4 & \\
\hline & BnaA03g46540D & 58.5 & & 65.8 & & 99.7 & & 63.7 & & 88.7 & \\
\hline & BnaC01g16110D & 58.6 & & 84.1 & & 63 & & 94.9 & & 62.4 & \\
\hline & BnaC07g38820D & 59 & & 66.2 & & 90.1 & & 63.6 & & 93.4 & \\
\hline Kelch & & At3g05420 & At5g27630 & Bra020582 & Bra001147 & Bra039439 & Bra040219 & Bol012774 & Bol001638 & Bol002733 & Bol034106 \\
\hline \multirow[t]{8}{*}{ motif } & AIS76194 & 90 & 75.7 & 75.6 & 96.5 & 90.6 & 89.7 & 76.3 & 90.4 & 90.4 & 95.9 \\
\hline & AIS76195 & 91.8 & 76.9 & 77.5 & 89.3 & 99.3 & 90.4 & 77.4 & 98.5 & 90.4 & 90.8 \\
\hline & AIS76196 & 90.1 & 75.5 & 75.3 & 89.5 & 90.3 & 98.2 & 75.3 & 90.4 & 100 & 90.3 \\
\hline & AIS76197 & 78.2 & 81.6 & 96.4 & 75.1 & 76.9 & 74.6 & 99.6 & 77.7 & 75.5 & 76.6 \\
\hline & AIS76198 & 77 & 81.2 & 97.6 & 73.5 & 76.8 & 74 & 95.4 & 77.1 & 74.3 & 75 \\
\hline & AIS76199 & 88.8 & 74.8 & 75 & 95.5 & 90 & 88.9 & 75.7 & 89.7 & 89.7 & 96.4 \\
\hline & AIS76200 & 91.5 & 76.5 & 77.4 & 89.2 & 99.3 & 90.1 & 77 & 98.2 & 90.6 & 90.7 \\
\hline & AIS76201 & 91 & 76 & 76.2 & 89.2 & 92.7 & 96.1 & 76.2 & 92.8 & 97.6 & 90.1 \\
\hline
\end{tabular}

Numbers in bold are the highest percentage of identity.

Phe-255 and Ile-280 were uncommon. Finally, the alignment of all four classes of ACBPs highlighted conserved amino acid residues, but only within the ACBD. Nevertheless, ACBD had some dissimilar amino acid sequences in each class of ACBP (see Figure S2, Additional file 2). These findings indicated ACBPs differed in domain structure, and BnACBPs and AtACBPs domain structure were very similar.

\section{Phylogenetic relationship of Brassicaceae ACBPs}

Analysis was carried out to highlight the phylogenetic relationships among Brassicaceae ACBP families. Five species belonging to Brassicaceae family and two Monocots species belonging to Poaceae family were used for the analysis. Each species could contain ACBPs belonging to the four classes, except for $A$. lyrata in which class II was missing. Sixty-four amino acid sequences

Table 3 Comparison of amino acid sequence identity in kelch motif BnACBPs. Numbers are in percent (\%)

\begin{tabular}{|c|c|c|c|c|c|c|}
\hline & BnaA02g31230D & BnaA03g29000D & BnaA05g31780D & BnaAnng02420D & BnaC02g39790D & BnaC03g34240D \\
\hline AIS76194 & 75 & 96.7 & 91 & 90.4 & 76 & 95.9 \\
\hline AlS76195 & 77.2 & 89.5 & 99.7 & 90.9 & 77.1 & 91.4 \\
\hline AIS76196 & 74.4 & 89.7 & 90.7 & 100 & 75.2 & 90.6 \\
\hline AIS76197 & 95.7 & 75.1 & 77.4 & 75.5 & 98.4 & 76.8 \\
\hline AIS76198 & 99.9 & 73.5 & 77.2 & 74.3 & 95.1 & 75.3 \\
\hline AlS76199 & 74.4 & 95.6 & 90.4 & 89.7 & 75.4 & 96.7 \\
\hline AIS76200 & 77.1 & 89.4 & 99.7 & 90.6 & 76.7 & 91.3 \\
\hline AIS76201 & 75.3 & 89.4 & 93.1 & 97.6 & 75.9 & 91 \\
\hline
\end{tabular}

Numbers in bold are the highest percentage of identity. 


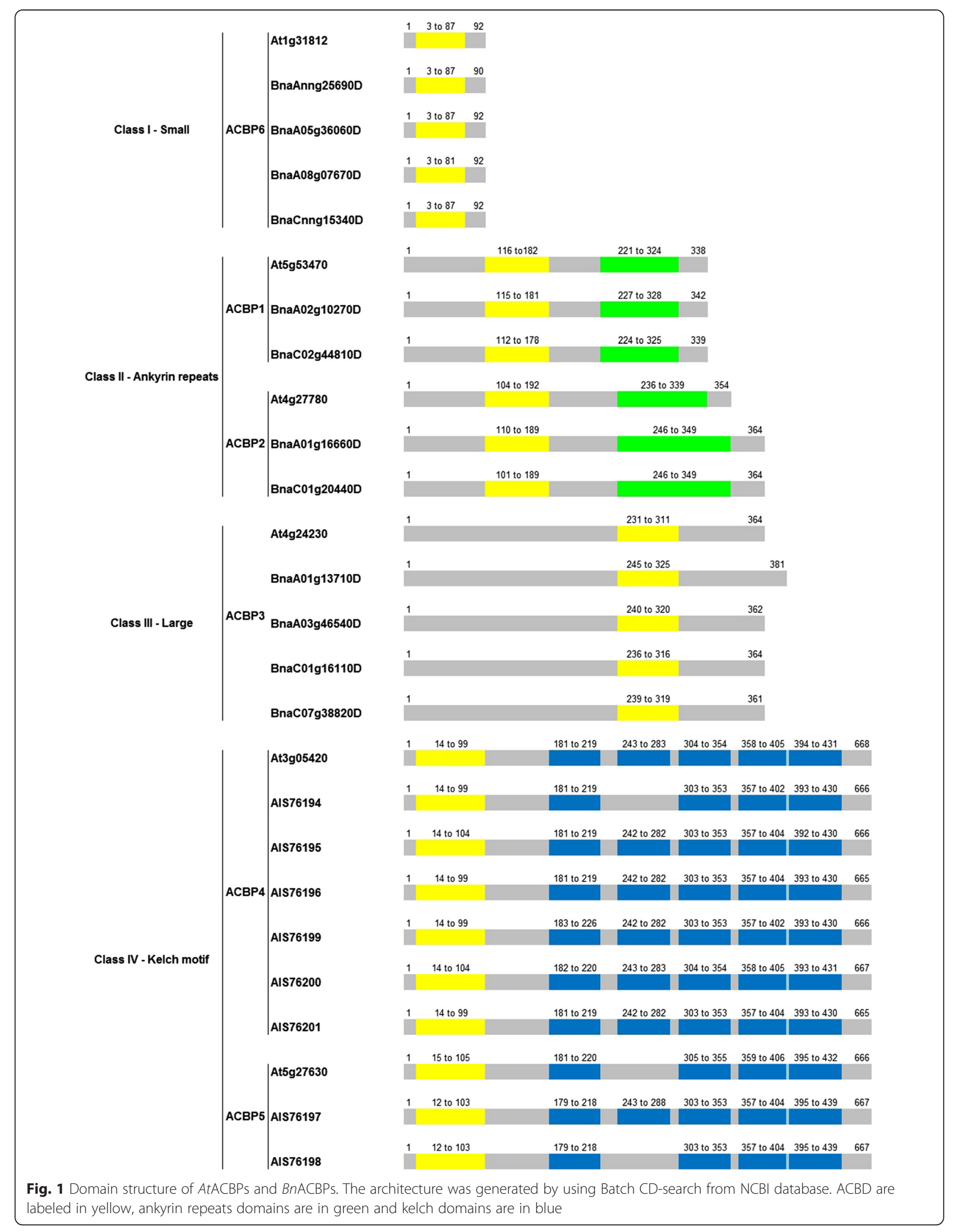


were involved in the analysis. The tree was rooted with Chlorella sp. (Aiu80187) and was inferred based on domain structure using Neighbor-joining (not shown) and Maximum likelihood (Fig. 2), topologies were very similar. The tree was divided into four major clusters corresponding to separate classes of ACBP. The topology of the tree related to each class of ACBP was almost uniform: Monocots (O. sativa and Z. mays) diverged before the Brassicaceae family and appeared to be distantly related with them. Obviously, class I was basal, but lacked bootstrap support (BS $<50$ \%). Class I ACBPs with 90 amino acids (Bra023206, Bol005980 and BnaAnng25690D) diverged separately from those with 92 amino acids. Class IV appeared as a well supported clade (BS $=99 \%$ ), it originated after the divergence of Class I. ACBP5 diverged before ACBP4; similarly in Class II, ACBP2 diverged before ACBP1. Class II was moderately supported (BS = $71 \%$ ). Class III, however, was weakly supported (BS = $63 \%)$. As in Class I, Class III shorter proteins (Bra019240, Bol042158, BnaA03g46540D and BnaC07g38820D) diverged separately from the longer ones. These results illustrated the evolutionary relationship of ACBPs, and their divergence leading to four distinct classes.

\section{Chromosome location of kelch motif BnACBPs}

In our previous analysis, eight copies of kelch motif $B n A C B P s$ were cloned and they corresponded to six copies of kelch motif BnACBPs found on the database. These six copies of kelch motif BnACBPs were located on six different chromosomes and they were associated to ancestors BrACBPs, BoACBPs and AtACBPs. Four maps were constructed. BnaA03g29000D (AIS76194) was located on A3, similar to parent Bra001147 (Fig. 3). BnaC03g34240D (AIS76199) was located on C3; parent Bol034106 was placed on Sca000040 (Fig. 3). To investigate where each chromosome was located on the scaffold, we used the blastp search of EnsemblPlants by EBI (http://plants.ensembl.org/) [23], with Bol034106 as query. Thus, Bol034106 is on Sca000040 of C3. As well, BnaAnng02420D (AIS76196) had homolog Bol002733 placed on Sca000378 of C1 (see Figure S3, Additional file 3). BnAnng02420D should have been found on $\mathrm{C} 1$, but obviously was located on an unspecified chromosome A. BnaA05g31780D (AIS76195/AIS76200) and parent Bra039439 were both placed on A5 (see Figure S4, Additional file 4). BnaC02g39790D (AIS76197) and homolog Bol012774 were both located on $\mathrm{C} 2$, whereas BnaA02g31230D (AIS76198) and Bra020582 were both placed on A2 (see Figure S5, Additional file 5). Chromosome location of BnACBP-AIS76201 could not be predicted, considering amino acid identity it could share with available genes on the database, it might be on a missing genomic contig. In this study, genes could be related in ascendant or descendant order but synteny was still preserved. Otherwise, one gene in A. thaliana might have two copies homolog on the same chromosome in Brassica, also one gene copy in $B$. rapa or $B$. oleracea might have two copies homolog in B. napus. For example, At3g05545 had two copies homolog to Bra039429 and Bra039430 on A5 (see Figure S4, Additional file 4). However, one copy of BrACBP or BoACBP had only one copy of BnACBP homolog on the related chromosome location. As predicted ACBP4, BnaA03g29000D (AIS76194), BnaC03g34240D (AIS76199), BnaA05g31780D (AIS76195/AIS76200) and BnaAnng02420D (AIS76196) were associated to homolog At3g05420 (AtACBP4) located on chromosome 3. As well, predicted ACBP5 BnaC02g39790D (AIS76197) and BnaA02g31230D (AIS76 198) were associated to At5g25630 (AtACBP5). These findings suggested $B$. napus inherited ACBP from $B$, rapa and $B$. oleracea, conserving their chromosome location.

\section{Discussion}

\section{ACBPs were conserved in Brassicaceae}

$B r A C B P s, B o A C B P s$ and BnACBPs were identified based on homology to AtACBPs. Their sizes were ranged from 90 to 667 amino acids, which were relatively close to those of AtACBPs with 92 to 668 amino acids [16]. Besides, eight copies of kelch motif ACBP were cloned from $B$. napus of a semi winter-type growing area in China. However, only six copies were found on the database given that two cloned copies (AIS76195 and AIS76200) shared the same amino acid sequence identity of $99.7 \%$ with one copy of the database (BnaA05g31780D), and one cloned copy (AIS76201) could only share its highest identity (97.6\%) with BnaAnng02420D, which already shared $100 \%$ of identity with AIS76196. This might be explained by the difference in plant materials. The Brassica napus French homozygous winter line 'Darmor-bzh' was used as reference for the genome sequencing [24]. The number of ACBP copies in B. napus obviously increased because $B$. napus inherited ACBP copies from its parents $B$. rapa and B. oleracea. Therefore, BnACBPs could be divided into four classes. BnACBPs shared an amino acid identity of $58.5 \%$ to $91.8 \%$ with AtACBPs, with the lowest rating in large ACBPs and the highest in the kelch motif ACBPs. Four small BnACBPs were acquired from the Genoscope Database. Earlier, six genes of about 90 amino acids in BnACBPs were identified, in which three genes were inherited from $B$. rapa and three genes were from $B$. oleracea [19]. The missing two copies corresponded to those inherited from B. oleracea (Bol027060 and Bol005980) given that they were not clearly specified from the database. Thus, small BnACBPs shared the $80.4 \%$ to $83.7 \%$ of amino acid sequence identity with AtACBPs, closely similar to previous finding in which the amino acid identity between small BnACBP and AtACBP was $84 \%[9,19]$. BnACBPs shared high amino 


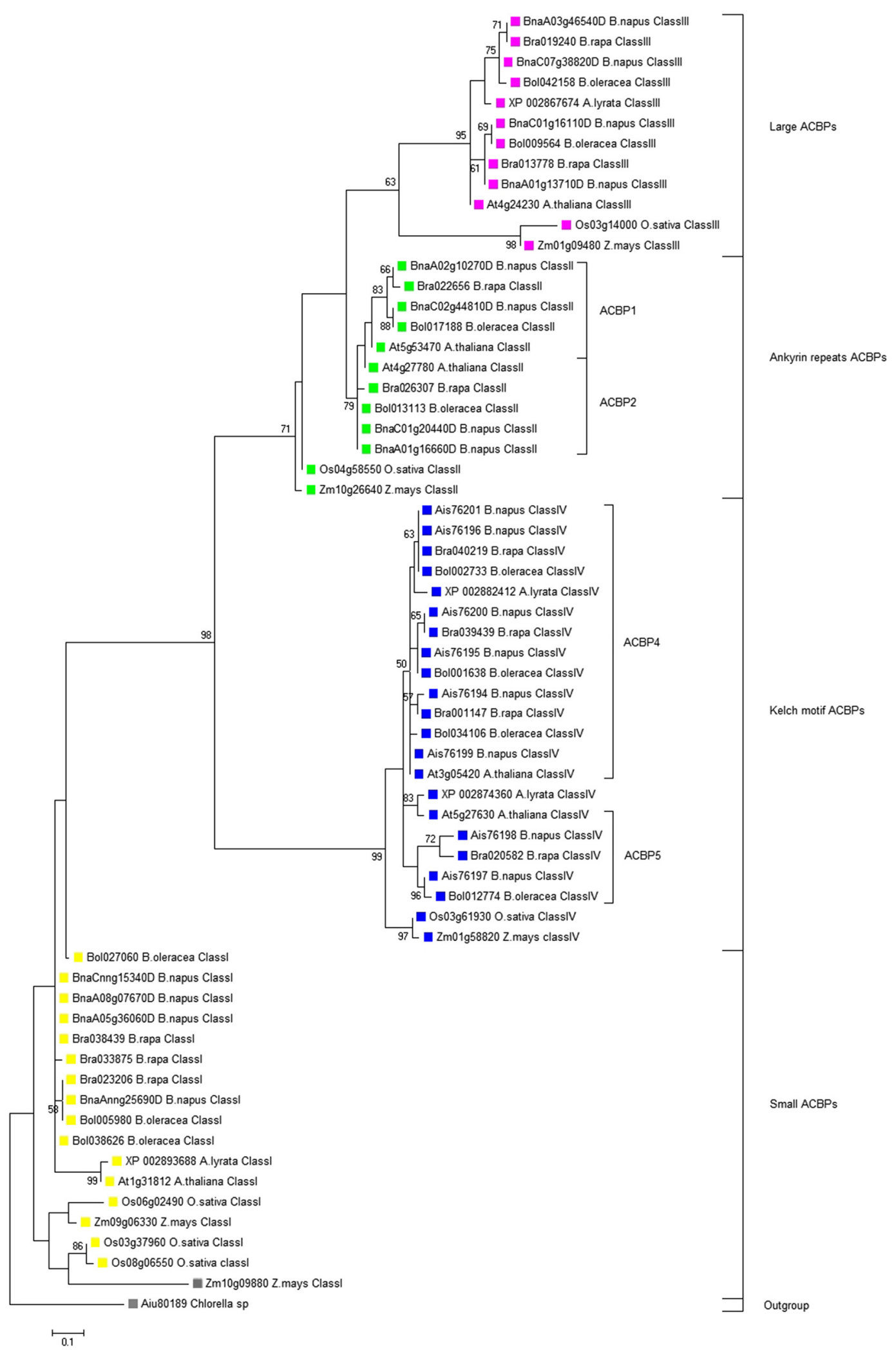

Fig. 2 Evolutionary relationships of land plant ACBPs. The evolutionary history was inferred by using the Maximum Likelihood method based on Equal input model. The analysis involved 64 amino acid sequences. Evolutionary analyses were conducted in MEGA6 


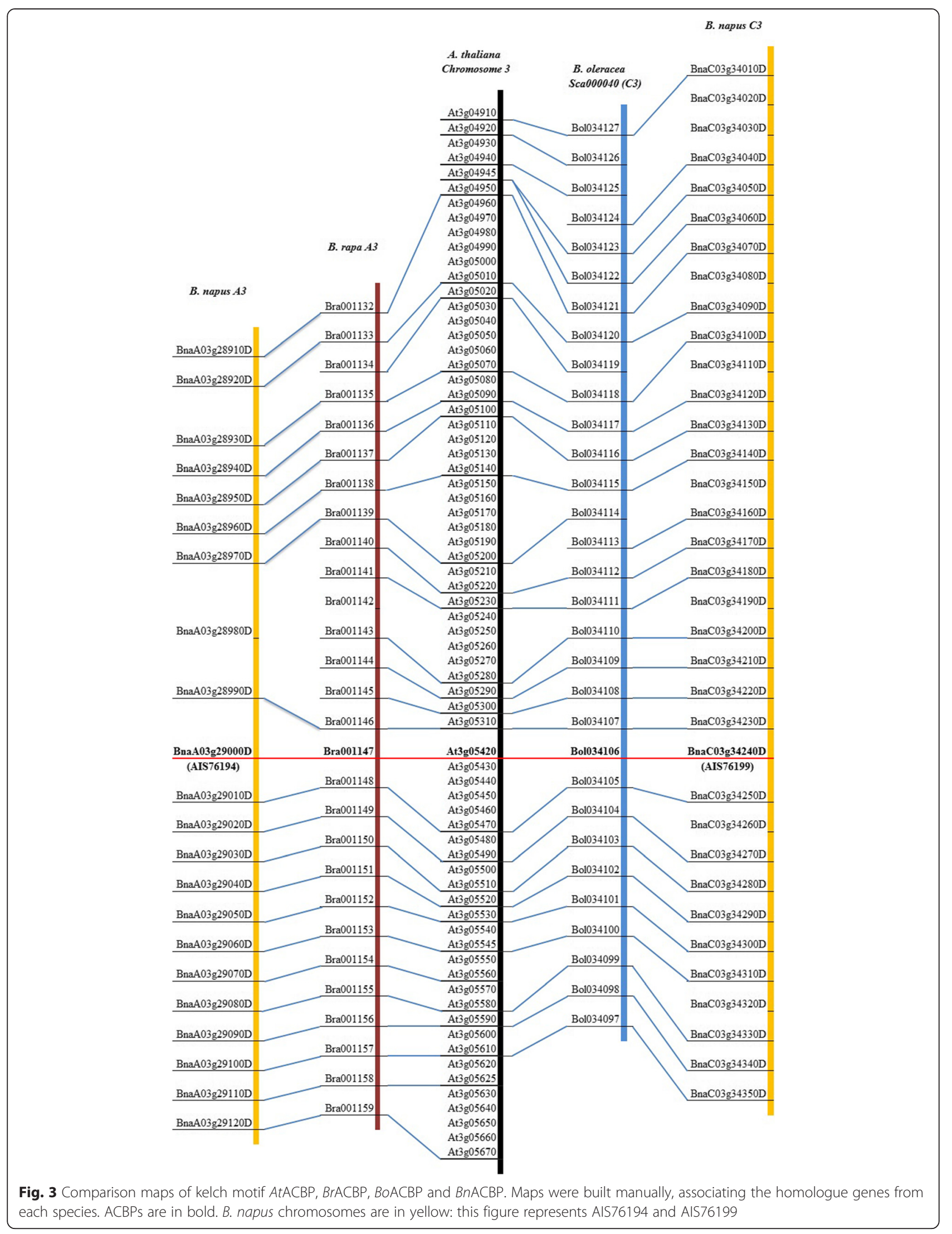


acid identity with AtACBPs, except for large ACBPs in which similarity was rather low compared to those of the other classes. Nevertheless, comparing large BnACBPs with homologues BrACBPs and BoACBPs exposed high amino acid sequence identity $93.4 \%$ to $99.7 \%$. Substantial mutations might have occurred during evolution resulting in a significant difference in amino acid sequence identity between these large AtACBPs and BnACBPs. Two classes of AtACBPs had two members: ankyrin repeats ACBPs and kelch motif ACBPs. Ankyrin repeats ACBPs could be subdivided in ACBP1 and ACBP2, they shared the $76.9 \%$ of identity [1]. Similarly, kelch motif AtACBPs included ACBP4 and ACBP5, which shared the $81.4 \%$ of identity [1]. Comparison in amino acid sequence identity allowed to define these subdivisions within the ankyrin repeats $B n A C B P s$ and the kelch motif BnACBPs. These findings affirm the conservation of ACBPs in Brassicaceae, with high amino acid sequence identity.

BnACBPs differed in domain structure, similar to AtACBPs Conserved domain in AtACBPs and BnACBPs were analyzed through batch CD-search of NCBI. ACBPs common domain structure was the acyl-coA binding domain (ACBD), which allowed them to fulfill their roles in binding acyl-coA esters with very high affinity [1-3]. $B n A C B P s$ structure conserved this ACBD but locations were different, as in AtACBPs. Moreover, in A. thaliana each class of ACBP had dissimilar affinity in binding acyl-coA as introduced previously. This dissimilarity might probably due to difference in amino acid sequence (see Figure S2, Additional file 2). Ankyrin repeats and kelch motif ACBPs differed from small and large ACBPs by the presence of protein-protein interaction site as additional domains $[13,14,18,21,25]$. One ankyrin domain was found in each protein. This domain of about 33 amino acid residues was involved in cell signals or regulation $[25,26]$. Four or five kelch motifs were found in our analysis; previous findings reported five kelch domains in AtACBPs [1, 10]. Kelch motif is a sequence of 44 to 55 amino acids in the protein; it is one of ancient and ordinary domains, and usually a group of four to seven motifs forms the kelch repeat domain [27, 28]. In our analysis, loss of one kelch motif (residue 242 to 283) was observed in At5g25630, AIS76194 and AIS76198. This loss might be explained with amino acid substitutions in this region of protein (see Figure S1, Additional file 1). Function or expression of protein might be different within ACBPs, in consequence of this loss. For instance, AtACBP4, which contained five kelch motifs were highly expressed in root whereas AtACBP5, which was demonstrated in our study to have only four kelch motifs, was expressed in shoots and mature, leaves [18]. These five kelch domains belonged to the Kelch 3 superfamily, but conserved amino acid residues were not identical, confirming the existence of multiple families of kelch domain [27, 28]. Ankyrin repeats and kelch motif domains could imply these ACBPs in many biological functions such as plant defense and stress responses [18, 22]: ankyrin repeats AtACBPs were involved in response to heavy metal accumulation [14] but also in tolerance of dry conditions [29]. Kelch motif AtACBP4 could act in response to accumulation of $\mathrm{Pb}$ (II) in root [30]. These findings confirm $B n A C B P s$ differ not only in size but also in domain structure.

\section{ACBPs evolved into four separate classes of diverse structure}

ACBPs from a selected group of Brassicaceae and Monocots were assembled for the phylogenetic analysis. To emphasize relationship among them, both NJ and ML tree were reconstructed, based on the conserved domain. Sixty-four ACBPs were involved, including ACBP of algae to root the tree. The presence of ACBPs in these species indicated their importance in biological function, and their existence before the divergence of land plants $[31,32]$. One class might have more than one copy due to duplication event resulting in dissimilar sequences [33]. In Monocots, class I contained two or three copies and the other classes contained only one copy, this shows that gene duplication occurred only in class I for this group [32]. A. lyrata Class II was missing. A.lyrata and $A$. thaliana are close enough that many traits are shared between them. Their divergence occurred about ten million years ago. A. lyrata genome is twice larger than that of $A$. thaliana. The missing Class II ACBPs in $A$. lyrata might have resulted from the insertions and deletion events that occurred in this evolution [34], leading to the loss of ankyrin repeats in A. lyrata ACBP. Monocots were distantly related to the Brassicaceae family, their divergence occurred earlier, during the evolution of land plants. In all ACBP classes, Brassica genera and Arabidopsis genera phylogenetic positions were close affirming that Brassica ACBPs shared common ancestry with Arabidopsis [35, 36]. The tree illustrated four major clusters corresponding to separate ACBP classes. Class I might be basal, since phylogenetic analysis with rice ACBPs suggested Class I might be ancestral [32], which is also accurate in our study. However, class I had inappropriate bootstrap support, which makes this hypothesis uncertain. Class I are single-domain proteins of about 90 amino acids, they were first cloned from $B$. napus [19]. Class I was demonstrated to be highly conserved in all species, not only in plants [37]. Class II and Class IV deviated separately after Class I divergence. In our analysis, ACBP1 and ACBP2 were distantly related, suggesting their early divergence, similarly to ACBP4 and ACBP5 of Class IV. Both Class II and Class IV are multi-domain proteins, which might be born from a pre- 
existing protein domain combination, since domains tend to combine within each other to generate new multi-domain proteins [38-40]. Class III originated after class II divergence; they had an N-terminal transmembrane domain as class II. Due to their phylogenetic position, class II and class III ACBPs were suggested to be functionally related [32]. ACBP is highly conserved in plants and paralogues born from a common ancestor [37]. ACBPs become larger with evolution [32]. This might explain the difference in size of ACBPs, smaller in the proposed ancestral Class I and larger in the other classes. In fact, protein domains as conserved in ACBPs are basic building blocks that define structure and function of proteins [40]; they have an independent evolution [41-43]. They originated from conglomerates of short polypeptide segments that fold together [44]. Duplication, divergence and recombination of domains generate proteins. New genes are mainly harbored from duplication and this could happen also for domains [42, 45]. Thus, evolution of ACBP by domain duplication and recombination might be possible. Duplication induced mutations explaining divergence; and recombination harbored new folds of new proteins with new functions that evolved from common ancestors [40, 46]. Consequently, protein domains change within sequence, structure and function but also in occurrence in the genomes of different organisms [43, 47]. Because duplication, convergence or divergence of protein domains [48-50], or formation of multi-domain proteins (as Class II and Class IV ACBPs) through domain combination [51, 52] are usual in protein evolution, this might have happened in evolutionary history of ACBP. Otherwise, ACBPs were demonstrated to show high evolutionary conservation in structure and function, precisely in basal cellular functions [31]. ACBPs were detected in different species: $30 \%$ of residues among them are conserved in the acylcoA binding domain (ACBD) [53]. Alignment of the four classes of ACBPs in our study confirmed ACBD as the only common domain; remaining amino acid residues had no similarities. Nevertheless, ACBDs showed some dissimilarity, which could explain diverse acyl-coA-binding preferences of different classes of ACBP [33, 43]. These findings confirm the high conservation of ACBPs in land plants and their diversity as consequences of evolution.

\section{$B$. napus inherited the kelch motif $A C B P s$ from $B$. rapa and B. oleracea}

Previously, our results demonstrated the conservation of ACBP in Brassicaceae. The kelch motif BnACBPs were demonstrated to conserve the same chromosome location as parents BrACBPs and BoACBPs, which homologues in A. thaliana were located on chromosomes 3 or 5 . In $A$. thaliana, chromosomes are subdivided into 24 blocks [54]. AtACBPs are located on block F of chromosome 3 and on block Q of chromosome 5. These blocks F and Q have corresponding blocks identified in B. napus [55] affirming their conservation in Brassica genera. Otherwise, gene loss and gene deletion possibly occurred within $B$. napus genome [24]. In our study, genes might appear to not have homolog in progenitors. This is the case of BnaA03g28980D and BnaC03g34150D which homolog was absent in A3 of B. rapa and C3 of B. oleracea, respectively (Fig. 3). Gene deletion might have occurred. Inversely, $B$. rapa and $B$. oleracea might not have homologues in B. napus. This is the case of Bra001142 and Bol034126 (Fig. 3), where gene loss might have occurred. Besides, genes could be present in A. thaliana but lacking in Brassica genera or inversely absent in $A$. thaliana but present in Brassica genera (case of Bol034113 on Fig. 3), as it was demonstrated that Brassica genome showed mixed losses and insertions compared with $A$. thaliana [55, 56]. Lastly, BnaAnng02420D (AIS76196), which is the homologue of Bol002733, was not found on chromosome $C$ in $B$. napus, but on an unspecified chromosome A (see Figure S3, Additional file 3). BnaAnng02420D might be a converted gene, as many converted genes were found in B. napus genome [24]. However, the analyzing of neighbor genes showed that Bol002724 had homolog BnaCnng24690D on an unspecified chromosome C, Bol002722 had homolog BnaC03g46060D on C3 and Bol002738 had homolog BnaC05g33680D on C5, suggesting a misassembly of this region. These results confirm that synteny is preserved and kelch motif ACBPs are faithfully transferred to B. napus.

\section{Overview on the evolutionary history of Brassica genomes, based on ACBPs}

The allotetraploid B. napus (AC, $\mathrm{n}=19$ ) was synthesized from hybridization between diploid parents $B$. rapa (A, $\mathrm{n}=10)$ and $B$. oleracea $(C, \mathrm{n}=9)$. The current study focused on ACBPs in B. napus, but their identification was made with homology based on $A$. thaliana, $B$. rapa and $B$. oleracea ACBPs, which could highlight the evolutionary history of Brassica genomes. ACBPs were conserved in Brassicaceae. The descendant line B. napus inherited 4 copies each of class I, class II and class III ACBPs, and 6 copies of kelch motif ACBPs (exclusive of results obtained from cloning). In total, 18 copies of ACBPs were inherited from parents $B$. rapa and B. oleracea and presented high amino acid sequence identity, similar structure and chromosome location with them. As 11 copies of ACBPs were found in each of B. rapa and B. oleracea (22 copies in total), four copies were lost in B. napus. They corresponded to two class I BoACBPs (Bol027060 and Bol005980), one class IV BoACBP (Bol001638) and one class IV BrACBP (Bra040219) (see Figure S6, Additional file 6). Besides, kelch motif ACBPs were 
faithfully transferred to $B$. napus, with six different chromosomal locations similar to parents $B$. rapa and B. oleracea. Genomic block $\mathrm{F}$ and $\mathrm{Q}$, which contain kelch motif AtACBPs were also in B. napus. The chromosome evolution of Brassica plants was studied through these genomic blocks, as reviewed by Cheng et al. [57]. They emphasized that whole genome triplication (WGT) promoted the diversification of Brassica plants. Genome comparison between A. thaliana and B. rapa or B. oleracea exposed this WGT event experienced by these two Brassica plants [58, 59]. In fact, Brassicaceae genomes are composed of 24 genomic blocks, the basic units of ancestral chromosome that reshuffled to generate the present day species. Then, three sets of 24 genomic blocks should exist in B. rapa and B. oleracea, which means 3 blocks $F$ and 3 blocks $Q$ each. This explains the increasing copies of kelch motifs ACBPs in B. rapa and B. oleracea (4 copies each). 3 blocks $F$ and 1 block Q, in each of $B$. rapa and B. oleracea, contain these kelch motif ACBP. However, 6 copies were inherited by B. napus, 2 copies were lost. These 6 conserved copies were on 4 blocks F (A3, A5, C1 and C3) and 2 blocks Q (A2 and C2). $B$. napus conserved these 6 genomic blocks from $B$. rapa and $B$. oleracea. These finding illustrate the evolutionary history of Brassica genomes, using ACBPs as reference.

\section{Conclusion}

Brassica napus ACBPs were identified and compared to ACBPs of relatives A. thaliana, B. rapa and B. oleracea. Their close similarity is not surprising, no major abnormality was found. ACBPs had multiple copies. As domain proteins, they originated from the same ancestor that experienced domain duplication and rearrangement to generate new proteins with different stucture. This study is a prelude for ACBP investigation in B. napus. Multiple copies were found, multiple interesting functions are expected for crop improvement purpose.

\section{Methods}

\section{Identification of BrACBPs and BoACBPs}

Identification of BrACBPS and BoACBPs was based on homology to $A$. thaliana ACBP proteins. A. thaliana ACBP (AtACBPs) were acquired from the Arabidopsis Information Resource - TAIR (http://www.arabidopsis.org) TAIR [60]. They were used as query to search for BrACBPs and BoACBPs in the Brassica database - BRAD (http://brassicadb.org) [61].

\section{Research of BnACBPs}

Kelch motif ACBPs were cloned from B. napus that were grown for 60 days in Huazhong University of Science and Technology, Wuhan, China (semi winter-type rapeseed growing area). The oil content of the material was about $50 \%$. We conducted RNA extraction from siliques, by using Trizol RNA isoplus (Takara). To synthesize the first cDNA strand, RevertAid First Strand cDNA Synthesis Kit by Thermo Scientific was used. Primers were designed based on kelch motif $B r A C B P s$ and BoACBPs sequences. Oligo7 software was operated to design these primers (sequences in Table S1, Additional file 7 ). The amplification was performed with KOD enzyme and then with ES Taq enzyme. The targeted genes were purified by CWBIO Gel extraction kit. They were then inserted into T-vector PMD19 by Takara Bio Inc. and integrated into $E$. coli DH5- $\alpha$. Genes from positive colonies were consequently sequenced. Vector NTI Advanced 11 software (Invitrogen Corporation) was used to align the obtained sequences with CDS of BrACBPs and $B o A C B P s$ to acquire CDS of BnACBPs, which were translated into protein sequences. Additionally, all four classes of BnACBPs were acquired from the CNSGenoscope database (http://www.genoscope.cns.fr/brassi canapus/) [24] with BrACBPs and BoACBPs as query.

\section{Comparison in amino acid sequence identity}

Two comparisons were made. The first one was between cloned kelch motif BnACBPs compared with those acquired from database, and the second one was between AtACBPs and BnACBPs. Amino acid sequence identity was calculated by using EMBOSS Needle (http://www.ebi. ac.uk/Tools/psa/emboss_needle/) [62].

\section{Conserved domain analysis}

Conserved domain in AtACBPs and BnACBPs were analyzed by using Batch CD-search tool in NCBI database, with E-value cut off 0.10 (http://www.ncbi.nlm.nih.gov/ Structure/bwrpsb/bwrpsb.cgi) [63].

\section{Phylogenic analysis based on domain}

ACBPs of selected species belonging to Brassicaceae family were used for the phylogenic analysis: Arabidopsis thaliana (Thale cress), Arabidopsis lyrata (Lyre-leaf rockcress), B. rapa (Field mustard), B. oleracea (Broccoli) and B. napus (Rape). Two Monocots were added to the tree: Oryza sativa (Rice) and Zea mays (Maize). Chlorella sp. was used to root the tree (see Table S2, Additional file 8). Proteins sequences were acquired from the NCBI database (http://ncbi.nlm.nih.gov). Alignment was performed with ClustalX software [64]. The phylogenic tree was inferred with Neighborhood Joining (NJ) [65], bootstrap 1000 and Maximum Likelihood method based on Equal input model [66], bootstrap 100, by using MEGA6 software [67].

\section{Chromosome location comparison of kelch motif AtACBPs, BrACBPs, BoACBPs and BnACBPs}

Comparison in chromosome location was based on kelch motif ACBPs. Hypothetical chromosome location of each 
BnACBPs was obtained from CNS-Genoscope database (http://www.genoscope.cns.fr/brassicanapus/). Chromosome locations of AtACBPs, BrACBPs and BoACBPs were obtained from TAIR (http://www.arabidopsis.org) and from BRAD (http://brassicadb.org). In order to know the arrangement of $B$. napus genes in the chromosome, genes neighbors of kelch motif BnACBPs were searched and associated to their respective homologues in $B$. rapa, B. oleracea and $A$. thaliana.

\section{Availability of supporting data}

The data set of Fig. 2 supporting the results of this article is available in the TreeBase (http://treebase.org/treebaseweb/home.html) repository, under the accession URL http://purl.org/phylo/treebase/phylows/study/TB2:S17727.

\section{Additional files}

Additional file 1: Figure S1. Alignment of kelch domains in AtACBPs and BnACBPs. I, II, III, IV and $V$ are the order of kelch domain on ACBPS. Highly conserved residues are in yellow, identical residues are in blue. Red framed residues (II) are putative altered residues.

Additional file 2: Figure S2. Alignment of the four classes of AtACBPs and BnACBPs. ACBD are framed in yellow, conserved residues are noticed. Ankyrin domains are framed in green, and kelch domain in blue.

Additional file 3: Figure S3. Comparison map of kelch motif AtACBP, BrACBP, BOACBP and BnACBP: AlS76196.

Additional file 4: Figure S4. Comparison map of kelch motif AtACBP, BrACBP, BoACBP and BnACBP: AIS76195.

Additional file 5: Figure S5. Comparison map of kelch motif AtACBP, BrACBP, BOACBP and BnACBP: AIS76197 and AIS76198.

Additional file 6: Figure S6. Evolutionary history of the Brassica genomes, based on ACBPs. Homology relationships are illustrated for $B$. rapa (AA), B. oleracea (CC) and B. napus (AACC). Chromosome location are mentioned. Underlined genes were lost during evolution. The tree was build with NJ methods and MEGA 6.

Additional file 7: Table S1. Primer sequences used to clone kelch motif BnACBPs.

Additional file 8: Table S2. List of plants species used in phylogenetic analysis.

\section{Competing interests}

The authors declare that they have no competing interests.

\section{Authors' contributions}

Conceived and designed the experiments: ML. Performed the experiments: NHR, YY, LC. Analyzed the data: NHR, YY, LC, ML. Contributed reagents/ materials/analysis tools: ML. Contributed to the writing of the manuscript: NHR, ML. All authors read and approved the final manuscript.

\footnotetext{
Acknowledgments

The work was supported by the International cooperation in science and technology projects (2014DFA32210), the National Basic Research Program of China (2015CB150205), the scientific research project of Hubei Collaborative Innovation Center for the Characteristic Resources Exploitation of Dabie Mountains (2015TD03) and New Century Talents Support Program of the Ministry of Education of China (NCET110172).
}

Received: 25 November 2014 Accepted: 29 June 2015

Published online: 09 July 2015

\section{References}

1. Leung KC, Li HY, Mishra G, Chye ML. ACBP4 and ACBP5, novel Arabidopsis acyl-CoA-binding proteins with kelch motifs that bind oleoyl-CoA. Plant Mol Biol. 2004:55:297-309.

2. Leung KC, Li HY, Xiao S, Tse MH, Chye ML. Arabidopsis ACBP3 is an extracellularly targeted acyl-CoA-binding protein. Planta. 2006;223:871-81.

3. Rosendal J, Ertbjerg P, Knudsen J. Characterization of ligand binding to acyl-CoA-binding protein. Biochem J. 1993;290(Pt 2):321-6.

4. Huang $H$, Atshaves BP, Frolov A, Kier AB, Schroeder F. Acyl-coenzyme A binding protein expression alters liver fatty acyl-coenzyme A metabolism. Biochemistry. 2005;44:10282-97.

5. Knudsen J, Faergeman NJ, Skott H, Hummel R, Borsting C, Rose TM, et al. Yeast acyl-CoA-binding protein: acyl-CoA-binding affinity and effect on intracellular acyl-CoA pool size. Biochem J. 1994;302:479-85.

6. Schjerling CK, Hummel R, Hansen JK, Borsting C, Mikkelsen JM, Kristiansen K, et al. Disruption of the gene encoding the acyl-CoA-binding protein (ACB1) perturbs acyl-CoA metabolism in Saccharomyces cerevisiae. J Biol Chem. 1996;271:22514-21.

7. Chye ML, Huang BQ, Zee SY. Isolation of a gene encoding Arabidopsis membrane-associated acyl-CoA binding protein and immunolocalization of its gene product. Plant J. 1999;18:205-14.

8. Chye ML, Li HY, Yung MH. Single amino acid substitutions at the acyl-CoAbinding domain interrupt 14[C] palmitoyl-CoA binding of ACBP2, an Arabidopsis acyl-CoA binding protein with ankyrin repeats. Plant Mol Biol. 2000;44:711-21.

9. Engeseth NJ, Pacovsky RS, Newman T, Ohlrogge JB. Characterization of an acyl-CoA binding protein form Arabidopsis thaliana. Arch Biochem Biophys. 1996;331:55-62.

10. Xiao S, Chye ML. An Arabidopsis family of six acyl-CoA-binding proteins has three cytosolic members. Plant Physiol Biochem. 2009;47:479-84.

11. Chen QF, Xiao S, Chye ML. Overexpression of the Arabidopsis 10-kilodalton acyl-Coenzyme A-binding protein ACBP6 enhances freezing tolerance. Plant Physiol. 2008;148:304-15.

12. Yurchenko OP, Nykiforuk CL, Moloney MM, Stahl U, Banas A, Stymne S, et al. A 10-kDa acyl-CoA-binding protein (ACBP) from Brassica napus enhances acyl exchange between acyl-CoA and phosphatidylcholine. Plant Biotechnol J. 2009;7:602-10

13. Li HY, Chye ML. Arabidopsis acyl-CoA-binding protein ACBP2 interacts with an ethylene responsive element-binding protein, AtEBP, via its ankyrin repeats. Plant Mol Biol. 2004;54:233-43.

14. Gao W, Xiao S, Li HY, Tsao SW, Chye ML. Arabidopsis thaliana acyl-CoAbinding protein ACBP2 interacts with a heavy-metal-binding protein AtFP6. New Phytol. 2008;181:89-102.

15. Li HY, Chye ML. Membrane localization of Arabidopsis acyl-CoA binding protein ACBP2. Plant Mol Biol. 2003;51:483-92.

16. Xiao S, Li HY, Zhang JP, Chan SW, Chye ML. Arabidopsis acyl-CoA-binding proteins ACBP4 and ACBP5 are subcellularly localized to the cytosol and ACBP4 depletion affects membrane lipid composition. Plant Mol Biol. 2008;68:571-83.

17. Xiao S, Chen QF, Chye ML. Light-regulated Arabidopsis ACBP4 and ACBP5 encode cytosolic acyl-CoA-binding proteins that bind phosphatidylcholine and oleoyl-CoA ester. Plant Physiology Biochemistry. 2009:47:926-33.

18. Li HY, Xiao S, Chye ML. Ethylene- and pathogen-inducible Arabidopsis acyl-CoA-binding protein 4 interacts with an ethylene-responsive element binding protein. J Exp Bot. 2008;59:3997-4006.

19. Hills MJ, Dann R, Lydiate D, Sharpe A. Molecular cloning of a cDNA from Brassica napus $L$. for a homologue of acyl-CoA-binding protein. Plant Mol Biol. 1994:25:917-20.

20. Yurchenko O, Singer SD, Nykiforuk CL, Gidda S, Mullen RT, Moloney MM, et al. Production of a Brassica napus low molecular mass acyl-CoA-binding protein in Arabidopsis alters the acyl-CoA pool and acyl composition of oil in seeds. Plant Physiol. 2014;165(2):550-60

21. Gao W, Li HY, Xiao S, Chye ML. Acyl-CoA-binding protein 2 binds lysophospholipase 2 and lysoPC to promote tolerance to cadmium-induced oxidative stress in transgenic Arabidopsis. Plant J. 2010;62:989-1003.

22. Du ZY, Chye ML. Interactions between Arabidopsis acyl-CoA-binding proteins and their protein partners. Planta. 2013:238:239-45.

23. Cunningham F, Amode MR, Barrell D, Beal K, Billis K, Brent S, et al. Ensembl 2015. Nucleic Acids Res. 2015:43:662-9.

24. Chalhoub B, Denoeud F, Liu S, Parkin IAP, Tang H, Wang X, et al. Early allopolyploid evolution in the post-Neolithic Brassica napus oilseed genome. Science. 2014;345:950. 
25. Mosavi LK, Minor Jr DL, Peng ZY. Consensus-derived structural determinants of the ankyrin repeat motif. Proc Natl Acad Sci. 2002;99:16029-34.

26. Bork P. Hundreds of ankyrin-like repeats in functionally diverse proteins: Mobile modules that cross phyla horizontally? Proteins. 1993;17:363-74.

27. Xue F, Cooley L. Kelch encodes a component of intercellular bridges in Drosophila egg chambers. Cell. 1993;72:681-93.

28. Adams J, Kelso R, Cooley L. The kelch repeat superfamily of proteins: propellers of cell function. Cell biology. 2000;10:17-24.

29. Du ZY, Chen MX, Chen QF, Xiao S, Chye ML. Arabidopsis acyl-CoA-binding protein ACBP1 participates in the regulation of seed germination and seedling development. Plant J. 2013;74:294-309.

30. Du ZY, Chen MX, Chen QF, Gu JD, Chye ML. Expression of Arabidopsis acyl-CoA-binding proteins AtACBP1 and AtACBP4 confers Pb(II) accumulation in Brassica juncea roots. Plant, Cell and Environment. 2015;38:101-17.

31. Faergeman NJ, Wadum M, Feddersen S, Burton M, Kragelund BB, Knudsen J. Acyl-CoA-binding proteins; structural and functional conservation over 2000 MYA. Mol Cell Biochem. 2007;299:55-65

32. Meng W, Su YCF, Saunders RMK, Chye ML. The rice acyl-CoA-binding protein gene family: phylogeny, expression and functional analysis. New Phytol. 2011;189:1170-84

33. Zhang JZ. Evolution by gene duplication: an update. Trends in Ecology and Evolution. 2003;18:292-8.

34. Hu TT, Pattyn P, Bakker EG, Cao J, Cheng JF, Clark RM, et al. The Arabidopsis lyrata genome sequence and the basis of rapid genome size change. Nat Genet. 2011;43:476-81.

35. Lysak MA, Lexer C. Towards the era of comparative evolutionary genomics in Brassicaceae. Plant Syst Evol. 2006:259:175-98.

36. Lysak MA, Koch MA, Pecinka A, Schubert I. Chromosome triplication found across the tribe Brassicaceae. Genome Res. 2005:15:516-25.

37. Burton M, Rose TM, Faergeman NJ, Knudsen J. Evolution of the acyl-CoA binding protein (ACBP). Biochem J. 2005:392:299-307.

38. Apic G, Gough J, Teichmann SA. An Insight into Domain Combinations. Bioinformatics. 2001;17:S83-9.

39. Ye Y, Godzik A. Database searching by flexible protein structure alignment Protein Sci. 2004:13:1841-50.

40. Itoh M, Nacher JC, Kuma K, Goto S, Kanehisa M. Evolutionary history and functional implications of protein domains and their combinations in eukaryotes. Genome Biol. 2007;8:R121.

41. Ponting CP, Russell RR. The natural history of protein domains. Annual Review Of Biophysics And Biomolecular Structure. 2002;31:45-71.

42. Vogel C, Bashton M, Kerrison ND, Chothia C, Teichmann SA. Structure, function and evolution of multidomain proteins. Curr Opin Struct Biol. 2004;14:208-16.

43. Yang S, Bourne PE. The evolutionary history of protein domains viewed by species phylogeny. PLoS ONE. 2009;4, e8378.

44. Lupas AN, Ponting CP, Russel RB. On the evolution of protein folds: are similar motifs in different protein folds the result of convergence, insertion, or relics of an ancient peptide world? J Struct Biol. 2001;134:191-203.

45. Lynch M, Conery JS. The evolutionary fate and consequences of duplicate genes. Science. 2000:290(5494):1151-5.

46. Grishin NV. Fold change in evolution of protein structures. J Struct Biol. 2001:134:167-85.

47. Scheeff ED, Bourne PE. Structural evolution of the protein kinase-like superfamily. PLoS Comput Biol. 2005;1, e49.

48. Bjorklund AK, Ekman D, Elofsson A. Expansion of protein domain repeats. PLoS Comput Biol. 2006:2:959-70.

49. Gough J. Convergent evolution of domain architectures (is rare) Bioinformatics. 2005;21(8):1464-71.

50. Vogel C, Morea V. Duplication, divergence and formation of novel protein topologies. Bioessays. 2006;28:973-8.

51. Kummerfeld SK, Teichmann SA. Relative rates of gene fusion and fission in multi-domain proteins. Trends Genet. 2005;21:25-30.

52. Vogel C, Teichmann SA, Pereira-Leal J. The relationship between domain duplication and recombination. J Mol Biol. 2005;346:355-65.

53. Kragelund BB, Poulsen $\mathrm{K}$, Andersen KV, Baldursson T, Kroll JB, Neergard TB, et al. Conserved residues and their role in the structure, function, and stability of acyl-coenzyme A binding protein. Biochemistry. 1999;38:2386-94.

54. Schranz ME, Lysak MA, Mitchell-Olds T. The ABC's of comparative genomics in the Brassicaceae: building blocks of crucifer genomes. Elsevier, Trends in Plant Science. 2006;11(11):535-42
55. Parkin IA, Gulden SM, Sharpe AG, Lukens L, Trick M, Osborn TC, et al. Segmental structure of the Brassica napus genome based on comparative analysis with Arabidopsis thaliana. Genetics. 2005;171:765-81.

56. Rana D, van den Boogaart T, O'Neill CM, Hynes L, Bent E, Macpherson L, et al. Conservation of the microstructure of genome segments in Brassica napus and its diploid relatives. Plant J. 2004;40:725-33.

57. Cheng F, Wu J, Wang X. Genome triplication drove the diversification of Brassica plants. Horticulture Research. 2014;1:14024.

58. Wang $X$, Wang $H$, Wang J, Sun R, Wu J, Liu S, et al. The genome of the mesopolyploid crop species Brassica rapa. Nat Genet. 2011;43:1035-9.

59. Liu S, Liu Y, Yang X, Tong C, Edwards D, Parkin IAP, et al. The Brassica oleracea genome reveals the asymmetrical evolution of polyploid genomes. Nature Communication. 2014:5:3930.

60. Lamesch P, Berardini TZ, Li D, Swarbreck D, Wilks C, Sasidharan R, et al. The Arabidopsis Information Resource (TAIR): improved gene annotation and new tools. Nucleic Acids Research. 2011.

61. Cheng F, Liu S, Wu J, Fang L, Sun S, Liu B, et al. BRAD, the genetics and genomics database for Brassica plants. BMC Plant Biol. 2011;11:136.

62. Rice P, Longden I, Bleasby A. EMBOSS: the European Molecular Biology Open Software Suite. Trends Genet. 2000;16(6):276-7.

63. Marchler-Bauer A, Derbyshire MK, Gonzales NR, Lu S, Chitsaz F, Geer LY, et al. CDD: NCBI's conserved domain database. Nucleic Acids Res. 2015:43:222-6.

64. Higgins DG, Sharp PM. CLUSTAL: a package for performing multiple sequence alignment on a microcomputer. Gene. 1988;73:237-44.

65. Saitou N, Nei M. The neighbor-joining method: A new method for reconstructing phylogenic trees. Mol Biol Evol. 1987;4:406-25.

66. Tajima F, Nei M. Estimation of evolutionary distance between nucleotide sequences. Mol Biol Evol. 1984;1:269-85.

67. Tamura K, Stecher G, Peterson D, Filipski A, Kumar S. MEGA6: Molecular Evolutionary Genetics Analysis version 6.0. Mol Biol Evol. 2013;30:2725-9.

\section{Submit your next manuscript to BioMed Central and take full advantage of:}

- Convenient online submission

- Thorough peer review

- No space constraints or color figure charges

- Immediate publication on acceptance

- Inclusion in PubMed, CAS, Scopus and Google Scholar

- Research which is freely available for redistribution 